\title{
A narrative review of combined stereotactic ablative radiotherapy and immunotherapy in metastatic non-small cell lung cancer
}

\author{
Zarique Z. Akanda ${ }^{1}$, Paul J. Neeson ${ }^{2,3}$, Thomas John ${ }^{4}$, Stephen Barnett ${ }^{5,6}$, Gerard G. Hanna ${ }^{1,2}$, \\ Alistair Miller ${ }^{7}$, Ross Jennens ${ }^{4}$, Shankar Siva ${ }^{1,2}$ \\ ${ }^{1}$ Division of Radiation Oncology and Cancer Imaging, Peter MacCallum Cancer Centre, Melbourne, Australia; ${ }^{2}$ Sir Peter MacCallum Department \\ of Oncology, The University of Melbourne, Melbourne, Australia; ${ }^{3}$ Division of Cancer Research, Peter MacCallum Cancer Centre, Melbourne, \\ Australia; ${ }^{4}$ Division of Medical Oncology, Peter MacCallum Cancer Centre, Melbourne, Australia; ${ }^{5}$ Division of Thoracic Surgery, Austin Health, \\ Heidelberg, Australia; ${ }^{6}$ Austin Health Department of Surgery, The University of Melbourne, Melbourne, Australia; ${ }^{7}$ Division of Respiratory \\ Medicine, The Royal Melbourne Hospital, Melbourne, Australia \\ Contributions: (I) Conception and design: ZZ Akanda, S Siva; (II) Administrative Support: None; (III) Provision of study materials or patients: None; \\ (IV) Collection and assembly of data: ZZ Akanda; (V) Data analysis and interpretation: ZZ Akanda, S Siva; (VI) Manuscript writing: All authors; (VII) \\ Final approval of manuscript: All authors. \\ Correspondence to: Shankar Siva, PhD, MBBS, FRANZCR. Division of Radiation Oncology and Cancer Imaging, Peter MacCallum Cancer Centre, \\ 305 Grattan Street, Melbourne, VIC 3000, Australia. Email: Shankar.Siva@petermac.org.
}

\begin{abstract}
Immune checkpoint inhibitors (ICIs) have significantly improved overall survival (OS) in metastatic non-small cell lung cancer (m-NSCLC). However, not all patients with m-NSCLC benefit from ICIs, and resistance to ICIs is an emerging challenge. The tumour microenvironment (TME) is immunosuppressive, and provides a myriad of mechanisms to facilitate escape of cancer cells from immune surveillance. The TME may also dampen the response to ICIs by inhibiting $\mathrm{T}$ cell effector responses. The poor prognosis of m-NSCLC has led to investigation of ICIs combined with other treatments with the intention of modulating the TME and sensitizing tumours to the effects of ICIs. Stereotactic ablative radiotherapy (SABR) in combination with ICIs is an area of intense interest. SABR is thought to evoke a pro-immunogenic response in the TME, with the capacity to turn a "cold", unresponsive tumour to "hot" and receptive to ICI. In addition to improved local response, SABR is postulated to produce a heightened systemic immune response when compared to conventional radiotherapy (RT). Preclinical studies have demonstrated a synergistic effect of SABR + ICIs, and clinical studies in m-NSCLC showed safety and promising efficacy compared to systemic therapies alone. To optimize ICI + SABR, ICI choice, combinations, dosing and length of treatment, as well as sequencing of ICI + SABR all require further investigation. Appropriate sequencing may depend on the ICI(s) being utilized, with differing sites of metastases possibly eliciting differing immune responses. Single versus multisite radiation is controversial, whilst effects of irradiated tumour volume and nodal irradiation are increasingly recognized. Taken together, there is strong preclinical and biological rationale, with emerging clinical evidence, supporting the strategy of combining $\mathrm{SABR}+\mathrm{ICI}$ in $\mathrm{m}-\mathrm{NSCLC}$.
\end{abstract}

Keywords: Stereotactic body radiation therapy (SBRT); non-small cell lung cancer (NSCLC); immune checkpoint inhibitors (ICIs); toxicity; radioimmunotherapy

Submitted Oct 27, 2020. Accepted for publication Mar 12, 2021.

doi: $10.21037 /$ tlcr-20-1117

View this article at: http://dx.doi.org/10.21037/tlcr-20-1117 


\section{Introduction}

Non-small cell lung cancer (NSCLC) accounts for $85 \%$ of all lung cancers, and is the leading cause of cancer related mortality (1). Prior to the advent of immunotherapy, historically metastatic NSCLC (m-NSCLC) carried a poor prognosis with 5-year survival rates of approximately $6 \%$ (1). Fifty percent of patients have metastatic disease at diagnosis (1). Tumours have a variety of mechanisms for escaping immune surveillance including up regulation of immune checkpoint molecules (2). Immune checkpoint molecules act as regulators of the immune system; they suppress ongoing effector T-cell activation, thereby reducing the potential for autoimmunity following chronic antigen presentation (2). The advent of immune checkpoint inhibitors (ICIs) transformed the treatment landscape of m-NSCLC. Current approved ICIs target blockade of programmed cell death protein 1 (PD-1) or it's ligand (PDL1), as well as cytotoxic T-lymphocyte-associated protein 4 (CTLA4). ICIs have demonstrated significant overall survival (OS) benefits and progression free survival (PFS) benefits in m-NSCLC, while having a more tolerable toxicity profile than traditional chemotherapy regimens (3-12). While predictive biomarkers for ICI benefit are not perfect, currently tumours with high expression of PD-L1 ( $\geq 50 \%)$ appear to derive the best responses and survival improvements (13). However, this represents a limited subgroup of the population of patients with m-NSCLC. Tumour mutational burden (TMB) was also being explored as a biomarker for ICI response, but remains controversial (13).

Both primary and secondary resistance to ICI poses therapeutic challenges and precise mechanisms of resistance remain unclear. The importance of the immune system in primary and secondary resistance is being recognised for both an initial and sustained response to ICI. The tumour microenvironment (TME) may be driven towards an immunosuppressive phenotype, and tumour cells may undergo adaptive changes to evade immunoregulation (14). Given the poor prognosis of $\mathrm{m}$-NSCLC, and resistance to ICIs, alternative strategies are required to improve the number of patients who can derive benefit. Strategies to modulate the immune system, in combination with ICIs are of current interest.

Radiotherapy (RT) in combination with ICIs has emerged as an area of considerable interest in m-NSCLC. A retrospective analysis of the KEYNOTE 001 study revealed that patients who received $\mathrm{RT}$ prior to commencing
Pembrolizumab demonstrated significantly increased OS and PFS compared to patients who did not (15). RT, in particular stereotactic ablative radiotherapy (SABR) has many postulated systemic immunomodulatory effects. This will be the subject of this review. One outcome of SABR is the abscopal effect; where-by regression of nonirradiated tumours at sites distant from irradiated tumour is observed (16). In extreme and rare examples of the abscopal effect, complete tumour regression at distant sites has been observed (16). This phenomenon is likely a result of a systemic immune response elicited by RT. Preclinical data suggests that RT and ICIs work synergistically (17-19), whilst limited clinical trials have observed improved outcomes in m-NSCLC treated with SABR + ICIs (20-22) (Table 1). Several phase II and III trials examining SABR + ICIs (Table 2) in m-NSCLC are in progress. Many questions regarding optimal combination therapy remain unanswered. In this narrative review, we examine the current state of immunotherapy in m-NSCLC, the immumodulatory effects of RT, and the toxicity profile of RT/SABR + ICIs. Furthermore, we examine the impact of RT dosing, sequencing, and sites of radiation on combination therapy with ICIs. We present the following article in accordance with the Narrative Review reporting checklist (available at http://dx.doi.org/10.21037/tlcr-20-1117).

\section{Current state of play for ICI and NSCLC}

In the absence of targetable mutations, ICI's are a fundamental component of treatment of m-NSCLC and have emerged as a backbone of first line therapy. Most approved agents target blockade of PD1 and PD-L1: including Nivolumab, Pembrolizumab, Atezolizumab, and Durvalumab. Anti-CTLA4 therapy has not demonstrated benefit as monotherapy, and combination of anti-PD-1 and anti-CTLA4 has been the subject of a further clinical trial in m-NSCLC (11).

The benefits of ICIs were first demonstrated in two key randomized phase III trials (Checkmate 17 and 57), which established the anti-PD-1 antibody Nivolumab as a standard of care (SOC) in second line treatment of m-NSCLC. A significant OS benefit was demonstrated compared to Docetaxel for both squamous and non-squamous cell tumours, as well as PFS benefits in updated 3-year follow up data (3-5). The Keynote 10 trial demonstrated OS benefit of Pembrolizumab over Docetaxel as a second line agent, with high PD-L1 tumour expressing groups (PD-L1 $\geq 50 \%$ ) 
also demonstrating PFS over Docetaxel (6).

The success and improved toxicity profile of immunotherapy lead to the investigation of immunotherapy as monotherapy in treatment naïve patients with m-NSCLC. This was investigated in Keynote 024 and Keynote 042 trials, which examined the use of Pembrolizumab compared to chemotherapy. Keynote 24 specifically examined patients with $\mathrm{PD}-\mathrm{L} 1 \geq 50 \%$, with a near doubling of OS demonstrated in updated 3-year data $(7,8)$. This durability in survival was paired with a decreased toxicity profile compared to chemotherapy. The results further reinforced the importance of high PD-L1 status, as in Keynote 042, only this subgroup demonstrated a survival benefit (8).

The benefit of ICIs in high PD-L1 expressing tumours is well established. However, this subset comprises $~ 30 \%$ (13) of patients with m-NSCLC. Subsequent studies investigated the combination of immunotherapy and chemotherapy, as a means of sensitizing tumours to immunotherapy. This approach was examined in the Keynote 189 and Keynote 407 studies, which compared Pembrolizumab and chemotherapy to chemotherapy alone. The results of these studies showed OS benefit in all PD-L1 subgroups $(9,10)$ and established Pembrolizumab in combination with chemotherapy as first line SOC for both squamous and non-squamous NSCLC. A different approach examined in the checkmate 227, and more recent 9LA trials (NCT03215706), where the combination of Ipilimumab (an anti-CTLA4 inhibitor) with Nivolumab (an antiPD-1 inhibitor) was used either alone or in combination with chemotherapy and compared to chemotherapy alone. Survival benefit was demonstrated even in PD-L1 negative tumours, suggesting a potential role for dual agent immunotherapy (11). ICIs have also been explored in the adjuvant setting in the PACIFIC trial where the addition of Durvalumab after definitive chemoradiation for stage III disease resulted in significant OS and PFS benefit (12).

Modulation of the immune system to enhance the efficacy of ICIs has also been explored in m-NSCLC with agents other than chemotherapy. In the large phase III multi-center international IMpower 150 trial, Atezolizumab (A), an anti-PD-L1 agent; Bevacizumab (B), an antivascular endothelial growth factor agent (anti-VEGF); and combinations with chemotherapy (C) were compared (ABC $v s$. BC vs. AC). This investigation was unique as it was a recent trial that included patients with driver mutations (EGFR and ALK) where ICI has historically not been effective. Data of $\mathrm{ABC} v s . \mathrm{BC}$ is available, with the $\mathrm{ABC}$ arm demonstrating PFS benefits even in patients with driver 
Table 2 Current ongoing phase II and above trials examining immunotherapy and radiation combinations exclusively in m-NSCLC

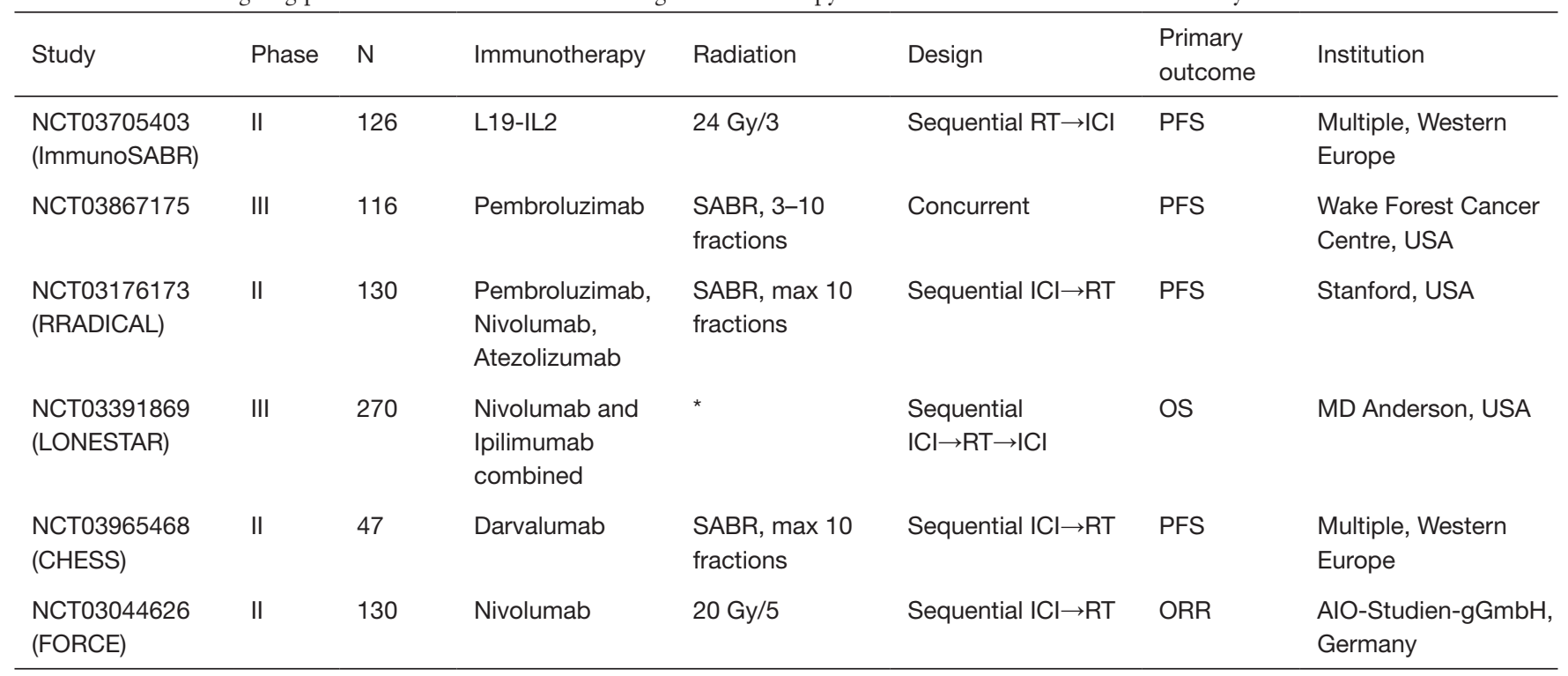

*, radiation treatment dose not available. m-NSCLC, metastatic non-small cell lung cancer; SABR, stereotactic ablative radiotherapy; ICI, immune checkpoint inhibitor; RT, radiotherapy; PFS, progression free survival; OS, overall survival.

mutations (23). Bevacizumab was postulated to inhibit the immunosuppressive effects of VEGF (24), and improve the efficacy of Atezolizumab.

\section{Mechanism of resistance}

Despite the success of immunotherapy in NSCLC, both primary and secondary resistance remains challenging. The broad principle of ICIs mechanism of action is promotion of the anti-tumour effects of T-cells. Thus, the success of ICIs is dependent on an effective anti-tumour immune response. Chen and Mellman have described the steps in this response, in what they have termed the cancer-immune cell cycle (24). An effective anti-tumour immune response requires adequate release of tumour associated antigens (TAAs) and subsequent antigen presentation for priming, activation and proliferation of tumour-specific T-cells in the tumour draining lymph node. Tumour-specific effector T-cells traffic to the tumour, where they attack tumour cells presenting cognate antigen and induce tumour cell death. The TME and adaptive responses by tumours can disrupt any step(s) in this process, and may limit the efficacy of ICIs.

A lack of immunogenicity and insufficient neo-antigens is thought to contribute to de novo resistance to ICIs in m-NSCLC. An analysis by Rizvi et al. demonstrated an improved PFS and overall response rate (ORR) in patients receiving Pembrolizumab with a higher TMB, irrespective of PD-L1 expression (25). Additionally, in the Checkmate 227 trial, patients with high TMB receiving combination ICIs demonstrated significantly improved PFS compared to chemotherapy alone, in contrast there was no benefit in patients with low TMB (11). However, no OS benefit was seen based on TMB (11). An analysis of relapsed NSCLC patients receiving ICIs demonstrated a loss of neoantigens (26). Additionally, tumours can undergo adaptive changes to evade the immune system through disruption of antigen presentation such as down regulation of major histocompatibility complex class I (MHC-I), and mutations of IFN-gamma signalling required for production of chemokines to traffic effector T-cells to sites of tumour (27). IFN-gamma induces tumour associated macrophages (TAMs) to secrete chemokine motif ligands (CXCL): CXCL9, CXCL10, and CXCL11, which traffic effector T-cells to tumour in response to the CXC3/ CXCL9-11 pathway (28). These factors can contribute to both primary resistance (patients who have no initial response to ICI) and secondary resistance (patients who are not able to sustain a response to ICI).

The TME itself dampens anti-tumour immune response through immunosuppressive cytokines and cells. Important immunosuppressive cells include myeloid derived suppressor cells (MDSC) and regulatory CD4+ T-cells (T-regs). Clinically, the presence of MDSC has been associated with poorer response to ICIs (29) as well as promotion of cancer 
survival and invasion (30). The TME is infiltrated by T-regs, which suppress effector T-cell functions by secreting inhibitory cytokines including IL-10, TGF- $\beta$, and IL-35. T-regs also express immune checkpoint molecules CTLA4, PD-1 and LAG-3 to dampen APC responses, sequester IL-2 and produce peri-cellular adenosine (31). All these mechanisms require local cell proximity. To enable this T-regs secrete chemokines CCL3 and CCL4 which attract CCR5+ effector T-cells (31). The presence of pre-existing CD8+ T-cells in the TME correlate with response to ICIs in m-NSCLC $(32,33)$. The role of tissue resident memory T-cells $\left(\mathrm{T}_{\mathrm{RM}}\right)$ in cancer immune surveillance is also being recognised (34). They are a group of non-circulating T-cells located in peripheral tissue and inhibit solid tumour growth locally. In particular CD8+ $\mathrm{T}_{\mathrm{RM}}$, which are characterized by co-expression of the integrin CD103+ (34). The presence of $\mathrm{CD} 8+\mathrm{CD} 103+\mathrm{T}_{\mathrm{RM}}$ have been associated with survival and enhanced tumour-infiltrating lymphocytes (TILS) in clinical isolates of NSCLC (35). However, the TME may make these cells dysfunctional (36). In addition to immune cells, there is growing evidence that non-immune stromal cells within the TME play an important role in ICI resistance. For example, recent studies by Dominguez et al. and Kieffer et al., demonstrated that cancer associated fibroblast gene signatures were significantly increased in NSCLC ICI nonresponders $(37,38)$. Since loss of neoantigens during clonal evolution, and pro-regulatory TME changes are established mechanisms of resistance, alternative therapeutic strategies (such as radiation) to reinvigorate the immune recognition should be considered.

\section{Immune responses to radiation}

The anti-tumour effects of radiation have traditionally been viewed as a local response to DNA damage, resulting in tumour necrosis, mitotic catastrophe, autophagy, apoptosis, and cell senescence (39). There is growing evidence of the ability of radiation to elicit a systemic immune response through engagement of the adaptive immune system. This concept is referred to as immunogenic cell death (ICD) (40). Radiation induced ICD ultimately results in a coordinated anti-tumour response by allowing for antigens to be presented for T-cell priming and eventual trafficking to tumour sites (see Figure 1).

Cell death from RT results in the release of danger associated molecular patterns (DAMPs) and liberation of TAAs. DAMPs facilitate the recruitment and maturation of antigen presenting cells (APCs), which subsequently results in the cross presentation and priming of effector T-cells in draining lymph nodes (41). Key DAMPs that have been described in ICD include calreticulin, ATP, and high mobility group box 1 (HMGB1) (42). Calreticulin is expressed on the surface of tumour cells as a stress response to cellular damage. It is highly prophagocytic, promoting the uptake of TAAs by APCs (43). In response to necrosis, the nuclear protein HMGB1 is released extracellularly and can bind toll-like receptor 4 on DCs. This induces DC maturation and trafficking to the tumour draining lymph node, where antigen is cross-presented for effective T-cell priming (44). Moreover, type I IFN is released after radiation, and plays a role in licensing DCs for improved priming of tumour specific T-cells in draining lymph nodes leading to tumour control (45). Additionally, radiation induces increased expression of MHC-I on tumour cells, allowing for effector T-cells to mediate anti-tumour effects through increased tumour cell recognition (46). The expression of MHC-I can be increased in a dose dependent manner to RT (46). As well as chemokine expression, radiation promotes extravasation and margination of immune cells through increased endothelial cell expression of intercellular adhesion molecules (I-CAMS), which are up regulated in response to inflammatory mediators from RT damage to tissues (47). I-CAMS are ligands for lymphocyte function-associated antigen 1 (LFA-1), an integrin found on trafficking T-cells (48). The upregulation of I-CAMS enable strong adhesion of effector T-cells to endothelium, and facilitates extravasation (48). Another pathway garnering interest is the stimulator of interferon gene (cGAS-STING) axis. Activation of this pathway results in the expression of type I IFN, which plays an important role for DC activation and effector T-cell priming $(45,49)$. Cytosolic double stranded DNA (dsDNA) is sensed by cGAS, which results in a signal cascade to activate the adaptor protein STING to induce type I IFN. Cellular damage from radiation provides a source of dsDNA to activate this pathway (50).

\section{Dose, fractionation, and immunogenicity}

The precise mechanisms whereby radiation induces immunogenicity are still being elucidated, however preclinical evidence to date suggests dose and fractionation schemes alter immunogenicity. Although the optimal dose and fractionation remain unclear, it is evident that ablative doses of radiation are more immunogenic than conventional fractionation (19,50-53). It is postulated that a dose between 10-12 Gy per fraction strikes a balance between suboptimal 


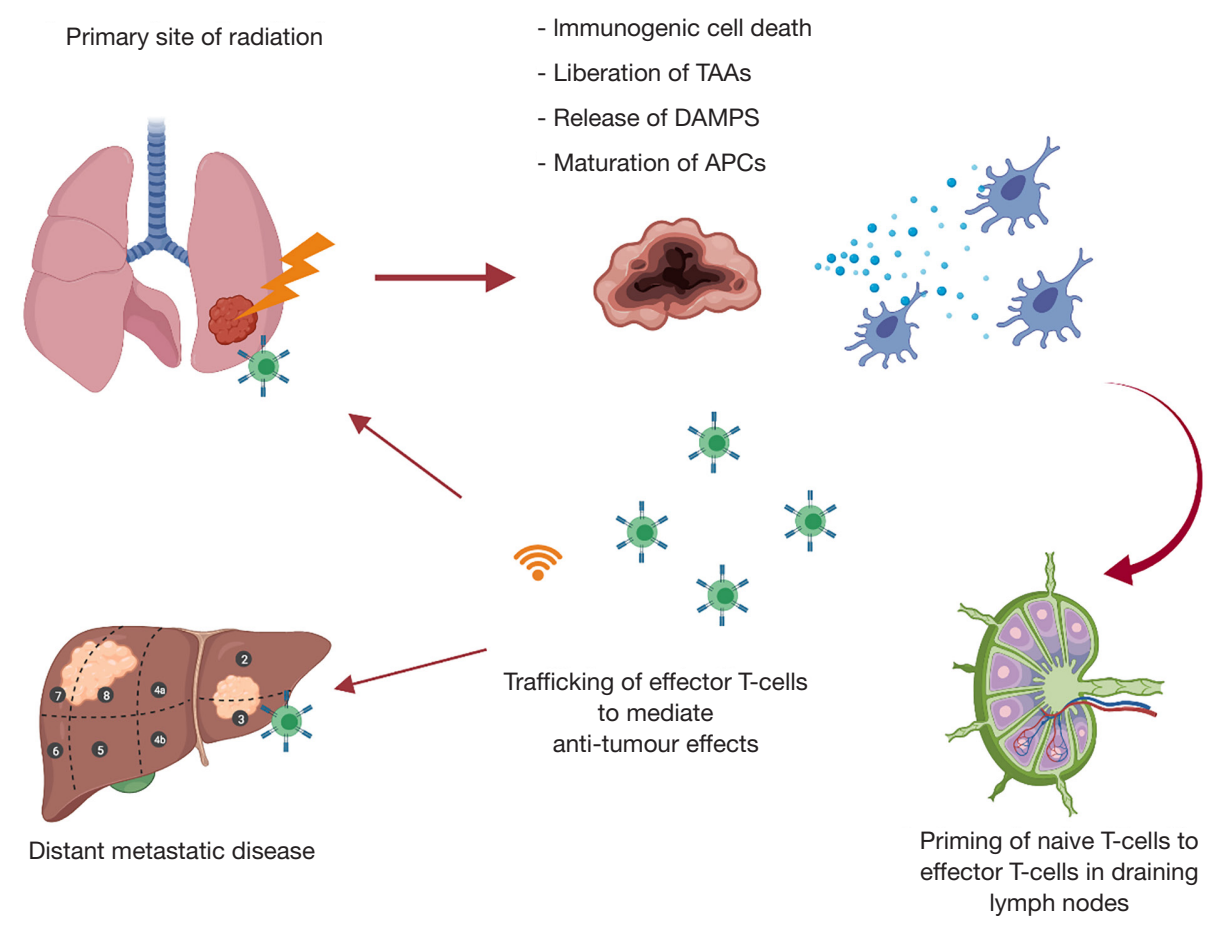

Figure 1 Illustration outlining mechanisms of a systemic immune response elicited from radiotherapy. Radiotherapy results in immunogenic cell death (ICD), subsequent release of danger associated molecular patterns (DAMPs), and liberation of tumour associated antigens (TAAs). DAMPS promote transcription of antigen presentation machinery, and maturation of antigen presenting cells (APCs). The release of TAAs allow for priming and activation of effector T-cells to mediate anti-tumour effects. DAMPS also result in the production of chemokines which traffic activated effector T-cells to mediate anti-tumour killing at both the primary irradiated site, and distal sites that share the same antigenic profile and secreting appropriate chemokines.

dosing, and potential immunosuppressive effects of highly ablative doses ( $>15 \mathrm{~Gy}$ ) such as enhancement of T-regs (54) and interference of the cGAS STING pathway $(55,56)$. It is notable, however, that high dose SABR as monotherapy is associated with the rarer form of abscopal effects with complete regression of distant sites, more readily than conventional fractionation $(16,57,58)$. In six reported cases of this phenomenon in NSCLC, four cases utilized a combination of SABR with ICIs (59). SABR lends itself as an attractive pairing with ICIs. Whether multi-fraction or single fraction SABR is optimal for engaging systemic responses in humans remains an unanswered question. Recent phase 1 and 2 clinical trials combining SABR, delivered in multiple fractions, with ICIs have shown an acceptable safety profile with promising efficacy (20-22).

\section{Sequencing of RT and ICI}

The optimal sequencing of RT and ICIs is yet to be definitively described in the clinic. However preclinical data suggests that ICIs are most effective when given concurrently, or in close sequence with RT to avoid delayed antigen presentation in an immune tolerant environment. The proposed mechanism of synergy is also dependent on ICI being utilized. Anti-PD-1/PD-L1 is thought to potentiate the activation of new T-cells and reinvigorate exhausted T-cells. Anti-CTLA4 acts in lymph nodes to inhibit T-regs, as well as rescuing co-stimulatory signals such that T-cells can respond to antigen presentation (60). Thus, these mechanisms theoretically act synergistically with RT induced T-cell activation and proliferation to create a more robust immune response.

Dovedi et al. compared RT with concurrent and a sequential delay of 7 days before administration of antiPD-L1 treatment. Anergy of effector T-cells and inferior survival benefit was observed in the sequential treatment group (17). This may be a result of ablation of newly proliferated and reinvigorated effector T-cells. Sequencing 
with RT and anti-CTLA4 has been studied in several animal models as well, with data suggesting that antiCTLA4 therapy is more effective when administered prior to RT $(61,62)$. In a study by Young et al., SABR (20 Gy $\times 1)$ was delivered with anti-CTLA4 administered 7 days prior, 1 day after, and 5 days after SABR. Survival benefit was best demonstrated with anti-CTLA4 delivered 7 days prior. These authors also examined the administration of anti-OX40 (a secondary co-stimulatory check point inhibitor) with the same stratification of RT sequence and found survival benefit to be greatest in the sequence of ICI 1 day after SABR (62). This further adds to the theory that optimal sequencing depends on the particular ICI being used. Sequencing with SABR and ICI (Pembrolizumab) is currently being explored specifically in $\mathrm{m}-\mathrm{NSCLC}$ in the SABRseq phase I trial (NCT03307759).

\section{Considerations for ideal targets}

A further question is whether RT to multiple sites in $\mathrm{m}-\mathrm{NSCLC}$ is more effective than to a single site. There is a strong biological rationale for irradiating multiple lesions, and modern RT techniques may allow for this to be safely delivered with minimal increase in resource utilization. As discussed previously, RT is immunogenic, releasing neoantigens, promoting antigen presentation, and creating infiltration of tumour by immune cells. An important consideration is the TME of any given site of disease, as local factors can influence the immune response elicited (63). A further consideration is the heterogeneity found within different metastatic lesions. That is to say the immune response elicited will only be efficacious for lesions that share the same antigenic profile, and sites secreting appropriate chemokines to traffic effector T-cells from the peripheral circulation. Theoretically, irradiation of multiple lesions should elicit a much more robust immune response, as more TAAs are accounted for, and a stronger systemic response may be mounted. This is an important factor to consider, as there is some evidence that not all sites of disease are equally immunogenic. Tang et al. investigated immunological correlates from patients who underwent RT + ICI. They found radiation of liver metastases resulted in an increased T-cell activation compared to pulmonary metastases (63). Furthermore, RT to multiple sites may decrease tumour bulk, potentially increasing efficacy of ICIs. This may not be accomplished with single site RT.

Although high level clinical evidence is limited, the results of clinical trials combining ICI $+\mathrm{RT}$ in the context of m-NSCLC may promote multisite radiation. In the phase 2 PEMBRO-RT trial, which delivered 24 Gy in 3 fractions, there was no statistically significant OS or PFS benefit to adding ablative RT; in that study only a single site of disease was radiated (22). However, a pooled analysis of two phase I/II trials examining single site SABR + Pembrolizumab vs. Pembrolizumab alone demonstrated OS benefit with SABR + Pembrolizumab (64). The phase 2 single arm study by Bauml et al., included only patients with oligometastatic disease and followed local ablation to all sites with delivery of Pembrolizumab. This resulted in a statistically significant increase in PFS to 19 months, compared to historical controls of 6.6 months (20). Luke et al. conducted a phase I study examining the safety of multi-site SABR (2-4 lesions) sequenced with Pembrolizumab in heavily pre-treated patients with solid organ malignancies including m-NSCLC. An acceptable safety profile was demonstrated, with less than $10 \%$ of patients experiencing dose-limiting toxicities, and an ORR of $13.5 \%$ was observed (65). Notably, this was less than the ORR observed in Formenti et al.'s study, which employed single site radiation and an ORR of $33 \%$ (21). The patient population in Luke et al.'s study, included treatment refractory patients, but offers a perspective on potential benefits of multi-site radiation in a clinical setting, even if abscopal effects are not as pronounced. Debulking of tumours may increase proliferating intratumoural T-cells (65) and reduce morbidity from metastatic disease.

A further consideration is the correlation between lymphopenia and decreased OS in many cancers, including m-NSCLC (66-69) and the possibility of increased consequential lymphopenia when multiple sites are irradiated. Radiation of large blood vessels, and lymphoid organs (such as bones and lymph nodes) may result in more persistent lymphopenia (70). Lymphocytes are generally radiosensitive, with varying degrees of sensitivities across subtypes, proliferative states, and location of T-cells, with lymphocytes in solid organs such as spleen and nodes being more radiosensitive (71). This is relevant clinically as priming of effector T-cells takes place in lymph nodes; tumour draining lymph nodes can be a target of elective irradiation. Preclinical studies have demonstrated the negative consequences of nodal radiation, as local control and trafficking of effector T-cells intratumourally may be diminished (72). More importantly this diminished response cannot be reversed by combination with ICI (62). In addition to site of radiation, larger volumes of RT may also worsen lymphopenia, as seen in NSCLC (73). These factors 
advocate for a lymphocyte sparing approach where possible.

\section{Intracranial combination therapy}

The efficacy of ICIs in metastases to the brain in $\mathrm{m}-\mathrm{NSCLC}$ is poorly described. These patients, especially when symptomatic are generally excluded from trials. Retrospective studies and limited phase I and II trials suggest safe toxicity profile of ICIs in treating brain metastases from NSCLC (74). Given that brain metastases are a poor prognostic factor, combination of stereotactic radiosurgery (SRS) and ICIs are being explored, in the hope of eliciting immunomodulation similar to that seen with extra cranial radiation. Retrospective studies however have shown limited efficacy. In a matched cohort study by Shepard et al. the combination of SRS + ICI did not result in benefit in OS, cranial PFS, or 1-year local control (75). From a toxicity perspective, there were no significant differences in rates of radiation necrosis, haemorrhage or oedema (75). Similar findings were also described in a study by Singh et al. which retrospectively compared SRS and anti-PD-1 to SRS and chemotherapy (76). The unique biology of the TME within the brain facilitates an immune privileged site, which theoretically may result in diminished immunomodulation. As described previously, the anti-tumour effects of ICIs are T-cell mediated. The blood brain barrier, and the brain itself include specialized cells such as astrocytes and glial cells that prevent cytotoxic cells from entering the brain further promoting tumour evasion (77). This reinforces the notion that not all sites of disease are equally immunogenic. The paucity of currently available clinical evidence mandates further investigation of intracranial radiation in combination with ICIs in m-NSCLC.

\section{Safety of combined radiation therapy and immunotherapy}

The immune-related adverse events (irAEs) are well described, including pneumonitis, fatigue, dermatitis, colitis, nephritis, and hepatitis; in severe cases reactions can be life threatening. The local and systemic toxicity of RT combined with ICIs could potentially preclude use of this combined approach. The safety profile of combination therapy is a subject of exploration. Retrospective analysis and limited published prospective trials indicate combination therapy is no more toxic than ICI monotherapy in m-NSCLC (78-81).
Sha et al. conducted a systematic review examining toxicity outcomes comparing ICI monotherapy to ICI + RT. Fifty-one studies (phase III and above), with over 15,000 patients were examined. Toxicities were comparable with $17.8 \%$ ( $n=1,442$; $95 \%$ CI: $12.0-24.5 \%)$ of patients experiencing grade $\geq 3$ toxicities with combination therapy (78). Conversely $22.3 \% \quad(n=13,956 ; 95 \%$ CI: $18.1-26.9 \%)$ of patients receiving ICI monotherapy, experienced grade $\geq 3$ toxicities (78). Additionally, there were no significant differences in toxicity when factoring in site of radiation (intra $v s$. extracranial) or the sequencing of RT (78).

In the context of m-NSCLC, available clinical evidence also supports the safety of combination therapy, including the use of SABR (and potential increased toxicities from ablative doses of RT). Randomized control trials where anti-PD-1/PD-L1 were used as monotherapy in advanced NSCLC reported grade $\geq 3$ irAE ranging from $7-16 \%(4,6,82)$. In Bauml et al. single arm study, grade 3 pneumonitis was only observed in $4 \%$ of patients and only $13 \%$ of patients experienced grade $\geq 3$ toxicities of any kind (20). The PEMBRO-RT trial also demonstrated an acceptable safety profile, with $20 \%$ of patients experiencing grade $\geq 3$ toxicities in SABR + ICI, and $30 \%$ in ICI alone (22). Moreover, no patients experienced grade $\geq 3$ colitis or pneumonitis in combination therapy (22). Chicas-Sett et al. systematically analysed prospective and retrospective studies, examining SABR + ICI outcomes in the setting of m-NSCLC. Again, similar toxicities of anti-PD-1/PD-L1 monotherapy to SABR-ICI were demonstrated (79).

Combination therapy with anti-CTLA4 demonstrated increased grade $\geq 3$ toxicities, with a weighted mean of $26.1 \%$ (67). The phase I/II trial by Formenti et al. is the only published prospective SABR + anti-CTLA4 (Ipilimumab) combination trial exclusively examining metastatic lung cancer. In this study grade $\geq 3$ toxicities were demonstrated in $38 \%$ of patients (21). High dose Ipilimumab (3 $\mathrm{mg} / \mathrm{kg})$ was administered, and toxicities observed may be more attributable to high dose ICI, than SABR in this combination. Lower doses of antiCTLA4 are have shown to be effective and less toxic, and are worth exploring with SABR. In Sha et al.'s systematic review, significantly more toxicity with anti-CTLA4 monotherapy was observed as compared to anti-PD-1/PDL1 monotherapy (78).

The toxicities of SABR with concurrent anti-CTLA4 were reviewed by Kroeze et al. Studies of concurrent 
SABR and anti-CTLA4 were limited to the treatment of brain metastases in the context of melanoma. The authors concluded the combination was safe, despite several studies demonstrating increased symptomatic radionecrosis with concurrent combination therapy (80). It is clear that the safety of combination RT + ICI will depend on the ICI being used and the dose and frequencies used. Further study is required to explore such combinations as they may add both immunogenicity through RT and maximize ICI benefit.

\section{Future speculations}

The technical aspects of delivering SABR with ICIs is a rapidly evolving field of exploration. Much of this exploration is driven by the desire to reduce toxicities as much as possible, while still attempting to induce an abscopal effect. Areas being explored include low dose wash radiation, partial volume irradiation with SABR, and FLASH RT.

RadScopal ${ }^{\mathrm{TM}}$ is a novel concept, which involves combining ICIs with ablative doses of RT to one lesion, while administering lower doses to other lesions. The rationale being that an ablative dose can prime the immune system with neoantigens, while lower doses can induce changes within the stroma of the TME to a more proinflammatory state amenable to ICI. Barsoumian et al. were able to demonstrate an abscopal response using this technique in murine models for both anti-PD-1 and anti-CTLA4 therapies (83). This theory remains in the preclinical phase of study but offers a potential avenue to reduce the toxicities of multiple sites of radiation. Another method being explored in the preclinical setting is the delivery of ultrahigh dose radiation in a rapid manner (FLASH). With this method, doses upwards of 40 Gy may be delivered over the span of one second (82). Although the mechanisms are unclear, preclinical models have shown reduced toxicity to healthy tissue, while maintaining local control of tumour (84). This ultrahigh dose rate therapy presents an entirely new biological approach, and the implication of this modality in combination with immunotherapy is not yet understood.

Partial radiation with SABR to tumour also represents another intriguing avenue of exploration in combination with ICIs, especially for large volume tumours. Tubin et al. investigated this approach in a clinical setting in 23 patients whose tumours were too large to SABR completely (85). Ten to 12 Gy per fraction was delivered in $1-3$ fractions to hypoxic areas of tumour bulk (85). The authors went on to examine what they defined as a bystander effect (regression in non-irradiated tumour bulk) and abscopal effects. A $96 \%$ response rate was observed in regards to bystander effect, and abscopal effects were observed for $52 \%$ of patients (85). Furthermore, no adverse toxicities were reported. The rationale for targeting hypoxic areas, is to overcome the downstream signalling cascades induced by hypoxia which promote evasion and tumour survival (86). This controversial but promising area of investigation is in need of further external validation.

\section{Conclusions}

ICIs have had a significant impact in the treatment of m-NSCLC with impressive OS and PFS compared to conventional chemotherapy, but their use is limited to selected patients, as innate and acquired resistance remains problematic. A robust immune response is thought necessary to maximize benefit from ICIs and in this context combination therapies are being explored. RT, particularly SABR is able to elicit a systemic immune response and is under investigation as combination therapy with ICI. Preclinical and clinical data suggests that ICI + SABR can work synergistically, but the mechanisms of synergy and the constitution of optimal combinations are still being elucidated. Studies combining ICI + SABR in m-NSCLC have demonstrated acceptable safety profiles compared to ICI monotherapy. Moreover, different sites of disease may have differing immunogenicity, with sites such as lymph node irradiation having a potentially negative impact on the immune response elicited. Multisite as compared to single site radiation still requires further investigation, but limited data suggest either approach is safe and potentially of interest as a strategy to move forward. Although many aspects require further investigation, recent phase II trials combining ICI + SABR in m-NSCLC have demonstrated reason for optimism, and appears to be an avenue worth exploring.

\section{Acknowledgments}

Funding: None.

\section{Footnote}

Provenance and Peer Review: This article was commissioned by the guest editor (Daniel Steinfort) for the series "Lung 
cancer and the immune system" published in Translational Lung Cancer Research. The article has undergone external peer review.

Reporting Checklist: The authors have completed the Narrative Review reporting checklist. Available at http:// dx.doi.org/10.21037/tlcr-20-1117

Peer Review File: Available at http://dx.doi.org/10.21037/ tlcr-20-1117

Conflicts of Interest: All authors have completed the ICMJE uniform disclosure form (available at http:// dx.doi.org/10.21037/tlcr-20-1117). The series "Lung cancer and the immune system" was commissioned by the editorial office without any funding or sponsorship. PJN reports grants from BMS, Roche Genentech, Allergan, and Compugen, outside the submitted work. TJ reports personal fees from Astra Zeneca, BMS, Novartis, MSD, Merck-Sorrono, Boehringer-Ingelheim, Roche, and Pfizer, outside the submitted work. GGH reports personal fees from Astra Zeneca, outside the submitted work. SS reports grants from Varian Industries, Merck-Sharp-Dohme, Astra Zeneca, and Bayer Pharmaceuticals, outside the submitted work. SS reports personal fees from Bristol Meyer Squibb, Astellas, Reflexion, Astra Zeneca, Roche, and Varian, outside the submitted work. The authors have no other conflicts of interest to declare.

Ethical Statement: The authors are accountable for all aspects of the work in ensuring that questions related to the accuracy or integrity of any part of the work are appropriately investigated and resolved.

Open Access Statement: This is an Open Access article distributed in accordance with the Creative Commons Attribution-NonCommercial-NoDerivs 4.0 International License (CC BY-NC-ND 4.0), which permits the noncommercial replication and distribution of the article with the strict proviso that no changes or edits are made and the original work is properly cited (including links to both the formal publication through the relevant DOI and the license). See: https://creativecommons.org/licenses/by-nc-nd/4.0/.

\section{References}

1. Siegel RL, Miller KD, Jemal A. Cancer statistics, 2019. CA Cancer J Clin 2019;69:7-34.
2. Wei SC, Duffy CR, Allison JP. Fundamental mechanisms of immune checkpoint blockade therapy. Cancer Discov 2018;8:1069-86.

3. Borghaei H, Paz-Ares L, Horn L, et al. Nivolumab versus Docetaxel in advanced non-squamous non-small cell lung cancer. N Engl J Med 2015;373:1627-39.

4. Brahmer J, Reckamp KL, Baas P, et al. Nivolumab versus Docetaxel in advanced squamous-cell non-small cell lung cancer. N Engl J Med 2015;373:123-35.

5. Font FE, Gettinger SE, Burgio MA, et al. Three-year follow-up from CheckMate 017/057: Nivolumab versus Docetaxel in patients with previously treated advanced non-small cell lung cancer (NSCLC). Ann Oncol 2017;28:v460-96.

6. Herbst RS, Baas P, Kim DW, et al. Pembrolizumab versus Docetaxel for previously treated, PD-L1-positive, advanced non-small cell lung cancer (KEYNOTE-010): a randomised controlled trial. Lancet 2016;387:1540-50.

7. Reck M, Rodríguez-Abreu D, Robinson AG, et al. Updated analysis of KEYNOTE-024: Pembrolizumab versus platinum-based chemotherapy for advanced nonsmall cell lung cancer with PD-L1 tumour proportion score of 50\% or greater. J Clin Oncol 2019;37:537-46.

8. Mok TSK, Wu YL, Kudaba I, et al. Pembrolizumab versus chemotherapy for previously untreated, PD-L1expressing, locally advanced or metastatic non-small cell lung cancer (KEYNOTE-042): a randomised, open-label, controlled, phase 3 trial. Lancet 2019;393:1819-30.

9. Gandhi L, Rodríguez-Abreu D, Gadgeel S, et al. Pembrolizumab plus chemotherapy in metastatic nonsmall cell lung cancer. N Engl J Med 2018;378:2078-92.

10. Paz-Ares L, Luft A, Vicente D, et al. Pembrolizumab plus chemotherapy for squamous non-small cell lung cancer. N Engl J Med 2018;379:2040-51.

11. Hellmann MD, Paz-Ares L, Bernabe Caro R, et al. Nivolumab plus Ipilimumab in advanced non-small cell lung cancer. N Engl J Med 2019;381:2020-31.

12. Antonia SJ, Villegas A, Daniel D, et al. Overall survival with Durvalumab after chemoradiotherapy in stage III NSCLC. N Engl J Med 2018;379:2342-50.

13. Lim SM, Hong MH, Kim HR. Immunotherapy for nonsmall cell lung cancer: current landscape and future perspectives. Immune Netw 2020;20:e10.

14. Jenkins RW, Barbie DA, Flaherty KT et al. Mechanisms of resistance to immune checkpoint inhibitors. Br J Cancer 2018;118:9-16.

15. Shaverdian N, Lisberg AE, Bornazyan K, et al. Previous radiotherapy and the clinical activity and toxicity of 
Pembrolizumab in the treatment of non-small cell lung cancer: a secondary analysis of the KEYNOTE-001 phase 1 trial. Lancet Oncol 2017;18:895-903.

16. Siva S, MacManus MP, Martin RF, et al. Abscopal effects of radiation therapy: a clinical review for the radiobiologist. Cancer Lett 2015;356:82-90.

17. Dovedi SJ, Adlard AL, Lipowska-Bhalla G, et al. Acquired resistance to fractionated radiotherapy can be overcome by concurrent PD-L1 blockade. Cancer Res 2014;74:5458-68.

18. Deng L, Huang L, Brunette B, et al. Irradiation and antiPD-L1 treatment synergistically promote anti-tumour immunity in mice. J Clin Invest 2014;124:687-95.

19. Dewan MZ, Galloway AE, Kawashima N, et al. Fractionated but not single dose radiotherapy induces an immune-mediated abscopal effect when combined with anti-CTLA4 antibody. Clin Cancer Res 2009;15:5379-88.

20. Bauml JM, Mick R, Ciunci C, et al. Pembrolizumab after completion of locally ablative therapy for oligometastatic non-small cell lung cancer: a phase 2 trial. JAMA Oncol 2019;5:1283-90.

21. Formenti SC, Rudqvist P, Golden E, et al. Radiotherapy induces responses of lung cancer to CTLA4 blockade. Nat Med 2018;24:1845-51.

22. Theelen WS, Peulen HM, Lalezari F, et al. Effect of Pembrolizumab after stereotactic body radiotherapy vs Pembrolizumab alone on tumour response in patients with advanced non-small cell lung cancer: results of the Pembro-RT phase 2 randomized clinical trial. JAMA Oncol 2019;5:1276-82.

23. Socinski MA, Jotte RM, Cappuzzo F, et al. Atezolizumab for first-line treatment of metastatic non-squamous NSCLC. N Engl J Med 2018;378:2288-301.

24. Chen DS, Mellman I. Elements of cancer immunity and the cancer-immune set point. Nature 2017;541:321-30.

25. Rizvi NA, Hellmann MD, Snyder A, et al. Cancer immunology. Mutational landscape determines sensitivity to PD-1 blockade in non-small cell lung cancer. Science 2015;348:124-8.

26. Anagnostou V, Smith K, Forde P, et al. Evolution of neoantigen landscape during immune checkpoint blockade in non-small cell lung cancer. Cancer Discov 2017;7:264-76

27. Bach EA, Aguet M, Schreiber RD. The IFN gamma receptor: a paradigm for cytokine receptor signaling. Annu Rev Immunol 1997;15:563-91.

28. House IG, Savas P, Lai J, et al. Macrophage-Derived CXCL9 and CXCL10 are required for antitumour immune responses following Immune checkpoint Bbockade. Clin Cancer Res 2020;26:487-504.

29. Meyer C, Cagnon L, Costa-Nunes CM, et al. Frequencies of circulating MDSC correlate with clinical outcome of melanoma patients treated with Ipilimumab. Cancer Immunol Immunother 2014;63:247-57.

30. Condamine T, Ramachandran I, Youn JI, et al. Regulation of tumour metastasis by myeloid-derived suppressor cells. Annu Rev Med 2015;66:97-110.

31. Josefowicz SZ, Lu LF, Rudensky AY. Regulatory T cells: mechanisms of differentiation and function. Annu Rev Immunol 2012;30:531-64.

32. Uryvaev A, Passhak M, Hershkovits D, et al. The role of tumour-infiltrating lymphocytes (TILs) as a predictive biomarker of response to anti-PD1 therapy in patients with metastatic non-small cell lung cancer or metastatic melanoma. Med Oncol 2018;35:25.

33. Herbst RS, Soria JC, Kowanetz M, et al. Predictive correlates of response to the anti-PD-L1 antibody MPDL3280A in cancer patients. Nature 2014;515:563-67.

34. Park SL, Gebhardt T, Mackay LK. Tissue-resident memory $\mathrm{T}$ cells in cancer immunosurveillance. Trends Immunol 2019;40:735-47.

35. Djenidi F, Adam J, Goubar A, et al. CD8+CD103+ tumour-infiltrating lymphocytes are tumour-specific tissue-resident memory $\mathrm{T}$ cells and a prognostic factor for survival in lung cancer patients. J Immunol 2015;194:3475-86.

36. Bengsch B, Ohtani T, Khan O, et al. Epigenomic-guided mass cytometry profiling reveals disease-specific features of exhausted CD8 T cells. Immunity 2018;48:1029-45.e5.

37. Dominguez CX, Muller S, Keerthivasan S, et al. SingleCell RNA Sequencing Reveals Stromal Evolution into LRRC15(+) Myofibroblasts as a Determinant of Patient Response to Cancer Immunotherapy. Cancer Discov 2020;10:232-53.

38. Kieffer Y, Hocine HR, Gentric G, et al. Single-cell analysis reveals fibroblast clusters linked to immunotherapy resistance in cancer. Cancer Discov 2020;10:1330-51.

39. Sia J, Szmyd R, Hau E, et al. Molecular mechanisms of radiation-induced cancer cell death: a primer. Front Cell Dev Biol 2020;8:41.

40. Golden EB, Apetoh L. Radiotherapy and immunogenic cell death. Semin Radiat Oncol 2015;25:11-7.

41. Pandolfi F, Altamura S, Frosali S, et al. Key role of DAMP in inflammation, cancer, and tissue repair. Clin Ther 2016;38:1017-28.

42. Sharabi AB, Lim M, DeWeese T, et al. Radiation and 
checkpoint blockade immunotherapy: radiosensitisation and potential mechanisms of synergy. Lancet Oncol 2015;16:e498-509.

43. Panaretakis T, Kepp O, Brockmeier U, et al. Mechanisms of pre-apoptotic calreticulin exposure in immunogenic cell death. EMBO J 2009;28:578-90.

44. Apetoh L, Ghiringhelli F, Tesniere A, et al. Toll-like receptor 4-dependent contribution of the immune system to anticancer chemotherapy and radiotherapy. Nat Med 2007;13:1050-59.

45. Burnette BC, Liang H, Lee Y, et al. The efficacy of radiotherapy relies upon induction of type I interferondependent innate and adaptive immunity. Cancer Res 2011;71:2488-96.

46. Reits EA, Hodge JW, Herberts CA, et al. Radiation modulates the peptide repertoire, enhances $\mathrm{MHC}$ class I expression, and induces successful antitumour immunotherapy. J Exp Med 2006;203:1259-71.

47. Ganss R, Ryschich E, Klar E, et al. Combination of T-cell therapy and trigger of inflammation induces remodeling of the vasculature and tumour eradication. Cancer Res 2002;62:1462-70.

48. Franciszkiewicz K, Le Floc'h A, Boutet M, et al. CD103 or LFA-1 engagement at the immune synapse between cytotoxic $T$ cells and tumour cells promotes maturation and regulates T-cell effector functions. Cancer Res 2013;73:617-28.

49. Deng L, Liang H, Xu M, et al. STING-dependent cytosolic DNA sensing promotes radiation-induced type I interferon-dependent antitumour immunity in immunogenic tumours. Immunity 2014;41:843-52.

50. Harding SM, Benci JL, Irianto J, et al. Mitotic progression following DNA damage enables pattern recognition within micronuclei. Nature 2017;548:466-70.

51. Lugade AA, Moran JP, Gerber SA, et al. Local radiation therapy of B16 melanoma tumours increases the generation of tumour antigen-specific effector cells that traffic to the tumour. J Immunol 2005;174:7516-23.

52. Morisada M, Clavijo PE, Moore E, et al. PD-1 blockade reverses adaptive immune resistance induced by highdose hypofractionated but not low-dose daily fractionated radiation. Oncoimmunology 2017;7:e1395996.

53. Schaue D, Ratikan JA, Iwamoto KS, et al. Maximizing tumour immunity with fractionated radiation. Int J Radiat Oncol Biol Phys 2012;83:1306-10.

54. Nishikawa H, Sakaguchi S. Regulatory T cells in tumour immunity. Int J Cancer 2010;127:759-67.

55. Vanpouille-Box C, Formenti SC, Demaria S. TREX1 dictates the immune fate of irradiated cancer cells. Oncoimmunology 2017;6:e1339857.

56. Vanpouille-Box C, Alard A, Aryankalayil MJ, et al. DNA exonuclease Trex1 regulates radiotherapy-induced tumour immunogenicity. Nat Commun 2017;8:15618.

57. Siva S, Callahan J, MacManus MP, et al. Abscopal effects after conventional and stereotactic lung irradiation of nonsmall cell lung cancer J Thorac Oncol 2013;8:e71-2.

58. Xing D, Siva S, Hanna GG. The abscopal effect of stereotactic radiotherapy and immunotherapy: fool's gold or el dorado? Clin Oncol (R Coll Radiol) 2019;31:432-43.

59. Bitran J. The abscopal effect exists in non-small cell lung cancer: a case report and review of the literature. Cureus 2019;11:e4118.

60. Seidel JA, Otsuka A, Kabashima K. Anti-PD-1 and antiCTLA4 therapies in cancer: mechanisms of action, efficacy, and limitations. Front Oncol 2018;8:86.

61. Twyman-Saint Victor C, Rech AJ, Maity A, et al. Radiation and dual checkpoint blockade activate non-redundant immune mechanisms in cancer. Nature 2015;520:373-7.

62. Young KH, Baird JR, Savage T, et al. Optimizing timing of immunotherapy Improves control of tumours by hypofractionated radiation therapy. PLoS One 2016;11:e0157164.

63. Tang C, Welsh JW, de Groot P, et al. Ipilimumab with stereotactic ablative radiation therapy: phase I results and immunologic correlates from peripheral T cells. Clin Cancer Res 2017;23:1388-96.

64. Welsh JW, Chen D, Baas P, et al. Radiotherapy to augment Pembrolizumab responses and outcomes in metastatic nonsmall cell lung cancer: pooled analysis of two randomized trials. J Clin Oncol 2020;38:abstr 9548.

65. Luke JJ, Lemons JM, Karrison TG, et al. Safety and clinical activity of Pembrolizumab and multisite stereotactic body radiotherapy in patients with advanced solid tumours. J Clin Oncol 2018;36:1611-8.

66. Zappasodi R, Merghoub T, Wolchok JD. Emerging concepts for immune checkpoint blockade-based combination therapies. Cancer Cell 2018;33:581-98.

67. Karantanos T, Karanika S, Seth B, et al. The absolute lymphocyte count can predict the overall survival of patients with non-small cell lung cancer on nivolumab: a clinical study. Clin Transl Oncol 2019;21:206-12.

68. Cho Y, Park S, Byun HK, et al. Impact of treatmentrelated lymphopenia on Immunotherapy for advanced non-small cell lung cancer. Int J Radiat Oncol Biol Phys 2019;105:1065-73.

69. Diehl A, Yarchoan M, Hopkins A, et al. Relationships 
between lymphocyte counts and treatment-related toxicities and clinical responses in patients with solid tumours treated with PD-1 checkpoint inhibitors. Oncotarget 2017;8:114268-80.

70. Ménétrier-Caux C, Ray-Coquard I, Blay J, et al. Lymphopenia in cancer patients and its effects on response to immunotherapy: an opportunity for combination with cytokines? J Immunother Cancer 2019;7:85.

71. Trowell OA et al. The sensitivity of lymphocytes to ionising radiation. J Pathol Bacteriol 1952;64:687-704.

72. Marciscano AE, Ghasemzadeh A, Nirschl TR, et al. Elective nodal irradiation attenuates the combinatorial efficacy of stereotactic radiation therapy and immunotherapy. Clin Cancer Res 2018;24:5058-71.

73. Venkatesulu BP, Mallick S, Lin SH, et al. A systematic review of the influence of radiation-induced lymphopenia on survival outcomes in solid tumours. Crit Rev Oncol Hematol 2018;123:42-51.

74. Vilariño N, Bruna J, Bosch-Barrera J, et al. Immunotherapy in NSCLC patients with brain metastases. Understanding brain tumour microenvironment and dissecting outcomes from immune checkpoint blockade in the clinic. Cancer Treat Rev 2020;89:102067.

75. Shepard MJ, Xu Z, Donahue J, et al. Stereotactic radiosurgery with and without checkpoint inhibition for patients with metastatic non-small cell lung cancer to the brain: a matched cohort study. J Neurosurg 2019:1-8.

76. Singh C, Qian JM, Yu JB, et al. Local tumour response and survival outcomes after combined stereotactic radiosurgery and immunotherapy in non-small cell lung cancer with brain metastases. J Neurosurg 2019;132:512-7.

77. Quail DF, Joyce JA. The microenvironmental landscape of brain tumours. Cancer Cell 2017;31:326-41.

78. Sha CM, Lehrer EJ, Hwang C, et al. Toxicity in combination immune checkpoint inhibitor and radiation therapy: a systematic review and meta-analysis. Radiother Oncol 2020;151:141-8.

79. Chicas-Sett R, Morales-Orue I, Castilla-Martinez J, et al. Stereotactic ablative Radiotherapy combined with immune checkpoint inhibitors reboots the immune response assisted by immunotherapy in metastatic lung cancer: a systematic review. Int J Mol Sci 2019;20:2173.

80. Kroeze SG, Fritz C, Hoyer M, et al. Toxicity of concurrent stereotactic radiotherapy and targeted therapy or immunotherapy: a systematic review. Cancer Treat Rev 2017;53:25-37.

81. Tian S, Switchenko JM, Buchwald ZS, et al. Lung stereotactic body radiation therapy and concurrent immunotherapy: a multicenter safety and toxicity analysis. Int J Radiat Oncol Biol Phys 2020;108:304-13.

82. Fehrenbacher L, Spira A, Ballinger M, et al. Atezolizumab versus Docetaxel for patients with previously treated non-small-cell lung cancer (POPLAR): a multicentre, open-label, phase 2 randomised controlled trial. Lancet 2016;387:1837-46.

83. Barsoumian HB, Younes AI, Ramapriyan R, et al. Low dose radiotherapy promotes immune-mediated antitumour responses. J Immunol 2019;202:abstr 136.11.

84. Wilson JD, Hammond EM, Higgins GS, et al. Ultra-high dose rate (FLASH) radiotherapy: silver bullet or fool's gold? Front Oncol 2020;9:1563.

85. Tubin S, Popper HH, Brcic L. Novel stereotactic body radiation therapy (SBRT)-based partial tumour irradiation targeting hypoxic segment of bulky tumours (SBRTPATHY): improvement of the radiotherapy outcome by exploiting the bystander and abscopal effects. Radiat Oncol 2019;14:21.

86. Rapisarda A, Melillo G. Overcoming disappointing results with antiangiogenic therapy by targeting hypoxia. Nat Rev Clin Oncol 2012;9:378-90.
Cite this article as: Akanda ZZ, Neeson PJ, John T, Barnett S, Hanna GG, Miller A, Jennens R, Siva S. A narrative review of combined stereotactic ablative radiotherapy and immunotherapy in metastatic non-small cell lung cancer. Transl Lung Cancer Res 2021;10(6):2766-2778. doi: 10.21037/tlcr-20-1117 